\title{
DJ-1 Mutations are Rare in a Swedish Parkinson Cohort
}

\author{
Anna Anvret ${ }^{1}$, Jeff G. Blackinton ${ }^{1,2}$, Marie Westerlund ${ }^{1}$, Caroline Ran ${ }^{1}$, Olof Sydow ${ }^{3}$, \\ Thomas Willows ${ }^{3}$, Anna Håkansson ${ }^{4}$, Hans Nissbrandt ${ }^{4}$ and Andrea Carmine Belin*, ${ }^{*}$ \\ ${ }^{I}$ Department of Neuroscience, Karolinska Institutet, Stockholm, Sweden \\ ${ }^{2}$ Department of Molecular Genetics and Microbiology, Duke University, Durham, NC, USA \\ ${ }^{3}$ Department of Neurology, Karolinska University Hospital, Stockholm, Sweden \\ ${ }^{4}$ Department of Pharmacology, Sahlgrenska Academy at the University of Gothenburg, Gothenburg, Sweden
}

\begin{abstract}
Mutations in the PARK7 gene, DJ-1, have been reported to cause early-onset and familial Parkinson's disease (PD). The function of DJ-1 and how it contributes to the development of the disease is not clear today, but several studies report that DJ-1 is responsive to oxidative stress and important for the maintenance of mitochondria. We have screened three coding regions of DJ-1 (exon 2, 5 and 7) in a Swedish Parkinson cohort. The Swedish PD material consisted of 67 patients with a self reported positive family history of PD and 77 patients with early-onset of disease ( $\leq 50$ years old). We detected two patients with the previously reported synonymous mutation, Ala167Ala (c.501A>G, rs71653621), in exon 7. No Ala167Ala carriers were identified among 213 neurologically healthy Swedish controls. Mechanisms by which the synonymous Ala167Ala mutation can have consequences are unknown. It may affect the mRNA stability, secondary structure of mRNA, synthesis, turnover, protein folding and function. We could show a $1.3 \%$ decrease in $D J-1$ mRNA folding energy in the $\mathrm{A}<\mathrm{G}$ substituted sequence compared to the wild type sequence in silico, suggesting a possible small effect of Ala167Ala on $D J-1$ gene function. This is the first report on an identified $D J-1$ mutation in Swedish PD patients. Our results, in combination with those of previous studies, strengthen the hypothesis that alterations in $D J-1$ are not a common cause of familial and early-onset PD world-wide.
\end{abstract}

Keywords: PARK7, mitochondria, mutation, oxidative stress.

\section{INTRODUCTION}

Parkinson's disease (PD) is a neurodegenerative disorder caused mainly by progressive loss of dopamine neurons in substantia nigra pars compacta. Pathways proposed to trigger onset of PD include oxidative stress and dysfunction of mitochondria [1]. The PARK7 gene, $D J-1$, has been reported to be responsive to oxidative stress and important for the maintenance of mitochondria $[2,3]$. DJ-1 was originally identified and described as an oncogene [4] and the 189 amino acid protein is highly conserved across species [5]. The DJ-1 protein is abundantly expressed throughout the body and in the normal human brain it is moderately expressed in neurons and in astrocytes throughout the CNS [6,7]. Oxidative modifications of DJ-1 have been reported in brain tissue from patients with sporadic PD and Alzheimer's disease compared to controls [8]. It has been suggested that DJ-1 protect neurons against oxidative stress by acting as a redox-dependent chaperone [9]. The protein is normally found in the cytoplasm and oxidative stress promotes relocation to mitochondria where it protects against mitochondrial toxins $[2,10]$. DJ-1 can bind RNA in an oxidative-dependent manner and the DJ-1/RNA complex dissociates after oxidative stress $[11,12]$. Structural studies of DJ-1 have suggested oxidation of Cysteine at residue 106 to be essential for DJ-1 to exert its

*Address correspondence to this author at the Department of Neuroscience, Retzius väg 8, B2:4, Karolinska Institutet, 17177 Stockholm, Sweden; Tel: +46-8-524 870 51; Fax: +46-8-323 742; E-mail: andrea.carmine.belin@ki.se full activities [3]. However it remains to be shown how oxidation of Cys106 leads to antioxidative and/or cytoprotective effects through conformational changes and how PD associated $D J-1$ mutations affect these processes.

In 2001 the PARK7 locus on chromosome 1p36 was linked to a family with autosomal recessive early-onset parkinsonism (AREP) in the Netherlands [13]. The following year the same locus was linked to a family with AREP in Italy [14]. Two mutations in $D J-1$ were later reported to cosegregate with disease, a $14 \mathrm{~kb}$ deletion in the Dutch PD family and a Leu166Pro substitution in the Italian PD family, and both lead to loss of gene function [5]. The DJ-1 mutations are recessively inherited and result in PD with earlyonset, overall mutations identified to date are rare, causing $\sim 1 \%$ of the PD cases world-wide and no mutations have been reported in Swedish PD cases [15]. In the Caucasian PD population the majority of $D J-1$ mutations leading to changes in the amino acid sequence have been found in exons 2, 5 and 7 [16]. We therefore sequenced these exons in a Swedish Parkinson cohort to search for known and new variable sites.

\section{MATERIAL AND METHODS}

\section{Subjects}

Exons 2, 5 and 7 and the exon/intron borders of $D J-1$ were sequenced in a Swedish PD material consisting of 144 individuals (mean age 64.3 years, $56.9 \%$ men). 77 of the PD patients had an early disease onset ( $\leq 50$ years old) and 67 of 
the patients had a self reported positive family history of PD in one or more first-, second- or third-degree relatives (15 of these patients had early-onset as well). 66 of the early-onset cases have previously been investigated for possible changes in copy numbers in exon $1,3,5$ and 7 of $D J-1$ although no aberrations were detected [17]. These patients were screened for Parkin point mutations and Parkin, $\alpha$-Synuclein, UCH-L1 and PINK1 exon copy number aberration as well. No known PD causing Parkin mutation or copy number aberration was found. Control subjects consisted of 213 neurologically healthy participants from the Stockholm area (mean age 75.8 years, $41.3 \%$ men). The material was obtained after informed oral and written consent and approval of the local ethics committee at Karolinska Institutet and University of Gothenburg. All PD subjects met the United Kingdom Parkinson's Disease Society Brain Bank Criteria for PD except that more than one affected relative was allowed [18]. DNA was extracted from blood according to standard protocol.

\section{Sequencing}

Samples from PD patients were analyzed with automated capillary sequencing. Polymerase chain reaction (PCR) was carried out using Taq DNA polymerase and the following primer sequences: exon 2 forward primer $5^{\prime}$-TCTCAGGG TTGCAATGAAAG-3'; reverse primer 5'-AAGCGTTAA ATGTGAGCAGTG-3', exon 5 forward primer $5^{\prime}$ AAATAGGTCAGAGAGCTTGTGG-3'; reverse primer 5'TCAAACCATCGAATGAAAGG-3' and exon 7 forward primer 5'-ACAGTGTTGGGTTTATATGCTG-3'; reverse primer 5'-GGACAGCGACTTCTGAACAC-3'. 40 cycles were run at $95^{\circ} \mathrm{C}$ for $45 \mathrm{~s}, 56^{\circ} \mathrm{C}$ for $40 \mathrm{~s}$ and $72^{\circ} \mathrm{C}$ for $1 \mathrm{~min}$ followed by $7 \mathrm{~min}$ terminal elongation at $72^{\circ} \mathrm{C}$. The PCR products were $337 \mathrm{bp}$ (exon 2), $258 \mathrm{bp}$ (exon 5) and $378 \mathrm{bp}$ (exon 7) of the DJ-1 gene. Purification of the amplified fragment was performed by using QIAquick ${ }^{\circledR}$ PCR Purification kit (Qiagen, Hilden, Germany). The isolated DNA fragments were sequenced using a DTCS kit followed by automated capillary gel electrophoresis (CEQ 2000 system, Beckman Coulter Inc., Fullerton, CA, USA), following the provider's instruction.

\section{Pyrosequencing}

To specifically screen neurologically healthy controls for the Ala167Ala mutation in exon 7 we used pyrosequencing
[19] and the following primer sequences; forward primer $5^{\prime}$ AGACGGCCTGATTCTTACAAGC-3'; reverse primer 5'GGCCTGTTTCTCTAAGTGATCG-3' and sequencing primer 5'-CTTCGAGTTTGCGC-3'. The reverse primer was biotinylated at the $5^{\prime}$-end. Positive controls for the Ala167Ala mutation were used in the pyrosequencing assay to guarantee accuracy of the method as well as water controls. PCR was carried out with Taq DNA polymerase to amplify a $175 \mathrm{bp}$ fragment. 45 cycles were run at $95^{\circ} \mathrm{C}$ for $20 \mathrm{~s}, 57^{\circ} \mathrm{C}$ for $20 \mathrm{~s}$ and $72^{\circ} \mathrm{C}$ for $30 \mathrm{~s}$ followed by $7 \mathrm{~min}$ terminal elongation at $72^{\circ} \mathrm{C}$, after which the biotinylated PCR product was immobilized on streptavidin-coated beads by mixing at $2500 \mathrm{rpm}$ for $10 \mathrm{~min}$ at room temperature according to manufacturer's instructions. The immobilized DNA template was then captured onto filter probes (PyroMark Vacuum Prep Tool, Biotage AB, Uppsala, Sweden). The filter probes were flushed with $70 \%$ ethanol, denaturation solution, washing buffer and the single-stranded template was annealed to a reverse sequencing primer at $80^{\circ} \mathrm{C}$ for 2 min followed by cooling to room temperature. All solutions used in sample preparation were prepared according to manufacturer's instructions (Biotage AB, Uppsala, Sweden). Samples were analyzed on an automated pyrosequencer using a PSQ 96 System together with single SNP Software and SNP Reagent Kits (Biotage AB, Uppsala, Sweden).

\section{Prediction of mRNA Secondary Structure}

To evaluate the possible effect of the identified Ala167Ala mutation on the $D J-1$ mRNA level, the secondary structure was predicted using the publicly available online software mfold version 3.2 [20,21]. Partial $D J-1$ mRNA sequences of 141 nucleotides including flanking sequences (70 nucleotides) on either side of the mutation were analyzed and compared to the wild type sequence.

\section{RESULTS}

In 144 PD cases we identified the synonymous mutation, Ala167Ala (c.501A>G, rs71653621), in exon 7 of $D J-1$ in two PD patients (Fig. 1 and Table 1). No Ala167Ala carriers were identified among the 213 neurologically healthy Swedish controls. To test whether the synonymous Ala167Ala substitution has a possible effect on the secondary structure of DJ-1 mRNA we performed a structure analysis in silico. Our results indicated a small decrease in mRNA folding en-

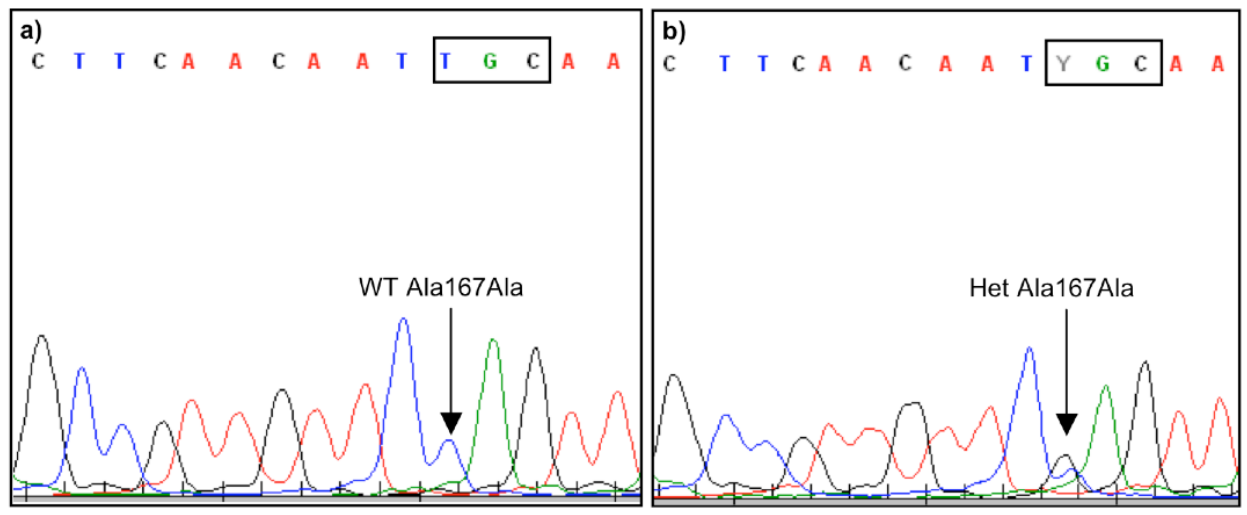

Fig. (1). Results from automated capillary sequencing of exon 7 in $D J-1$ including the Ala167Ala (c.501A>G, rs71653621) mutation using a reverse sequencing primer $(\mathrm{T}>\mathrm{C})$. a) A homozygous wild type (WT) T/T, Ala167Ala carrier and b) a heterozygous (Het) T/C, Ala167Ala carrier. 
Table 1. Patient Information on the Two Individuals with Parkinson's Disease (PD) Heterozygous for the DJ-1 Mutation Ala167Ala (c.501A>G, rs71653621) in Exon 7

\begin{tabular}{|c|c|c|c|c|c|}
\hline & Sex & Family history & Age at sampling & Age of onset & Brief history \\
\hline \hline CASE 1 & Female & Brother With PD & 71 & 69 & $\begin{array}{c}\text { Moderately advanced PD, ON/OFF with some motor fluctuations } \\
\text { but dominating non-motor symptoms in off. }\end{array}$ \\
\hline CASE 2 & Male & No known heredity & 65 & 48 & $\begin{array}{c}\text { Benign course. Minimal motor fluctuations but walking unassisted } \\
\text { after 24 years disease. Diagnosed with Alzheimer's disease as well } \\
\text { at age 66. }\end{array}$ \\
\hline
\end{tabular}

Table 2. In Silico mRNA Folding Energy (kcal/mol) for the Wild Type (WT) and Mutated (MUT) Ala167Ala DJ-1 Sequences

\begin{tabular}{|c|c|c|}
\hline \multirow{2}{*}{ Individual structures } & \multicolumn{2}{|c|}{ Minimum free energy (kcal/mol) } \\
\cline { 2 - 3 } & WT Ala167Ala & 38.80 \\
\hline \hline Structure 1 & 39.10 & 38.60 \\
\hline Structure 2 & 38.90 & 37.70 \\
\hline Structure 3 & 38.00 & 37.00 \\
\hline Structure 4 & 37.30 & 37.00 \\
\hline Structure 5 & - & 37.82 \\
\hline Average & 38.33 & \\
\hline
\end{tabular}

ergy (1.3\%) in the $\mathrm{A}>\mathrm{G}$ substituted sequence compared to the wild type sequence (Table 2 ).

\section{DISCUSSION}

The number of $D J-1$ mutations described today is low and they account for only a few PD cases in different populations [16]. In the present study, we have searched for mutations in exon 2, 5 and 7 and in the exon/intron borders of $D J$ 1 , since variations have been reported in these regions [22]. We detected a heterozygote Ala167Ala mutation in two out of 144 Swedish PD patients, but no Ala167Ala carriers were identified among the 213 neurologically healthy controls. This is the first report on $D J-1$ mutations identified in a Swedish PD material. The Ala167Ala mutation was previously identified in a PD patient (homozygote carrier) from the United Kingdom and in a patient from a North American material with Caucasian/Hispanic origin (heterozygote carrier) $[22,23]$. One of the Swedish Ala167Ala carriers was diagnosed with Alzheimer's disease as well (Table 1), which is in agreement with the reports that $D J-1$ has been implicated in Alzheimer's disease [8]. Mechanisms by which the synonymous Ala167Ala mutation may have consequences on the protein level and predispose to disease are not well understood. The DJ-1 protein has been suggested to act in multiple pathways and hypothesized that loss of the protective function of DJ-1 in dopaminergic neurons can lead to neurodegeneration [24]. Several possibilities have been suggested for synonymous mutations including alterations of mRNA stability, secondary structure, transcriptional activity, or changes in protein synthesis, folding, levels, turnover and/or function [25]. We found a small decrease in mRNA folding energy (1.3\%) comparing the mutated Ala167Ala sequence with the wild type sequence, suggesting a possible effect of Ala167Ala on DJ-1 gene function [26]. However, no strong conclusions can be drawn from the artificial conditions used for modeling secondary structures in silico.
The frequency of $D J-1$ mutations has been reported to vary among PD populations with different ethnic origin, Caucasian: $0.91 \%$, Asian: $3.03 \%$, Arab: $0.74 \%$ and Ashkenazi Jews: $1.96 \%$ and copy number variations in $D J-1$ have so far only been reported in the Caucasian PD population $(0.54 \%)$ [16]. We found the Ala167Ala mutation in two PD patients in our Swedish cohort, but no other genetic variant in any of the three investigated exons. The observed $D J-1$ mutation frequency $(1.4 \%)$ is in agreement with findings in other Caucasian PD populations.

\section{CONCLUSION}

This is the first report on a $D J-1$ mutation identified in Swedish PD patients. Based on the results of the screening in our Swedish cohort as well as the results from other studies, it appears clear that mutations in $D J-1$ are a rare cause of familial and early-onset PD world-wide.

\section{ACKNOWLEDGMENTS}

We would like to thank Professor Laura Fratiglioni for providing us with control samples from the Swedish National Study on Aging and Care in Kungsholmen (SNAC-K) project and Doctor Fengqing Xiang for excellent technical assistance. The study was supported by the Swedish Research Council, the Swedish Brain Foundation, Karolinska Institutet Funds, the Swedish Parkinson Foundation, Swedish Brain Power and Åhlen's Foundation.

\section{REFERENCES}

[1] Leroy E, Boyer R, Auburger G, et al. The ubiquitin pathway in Parkinson's disease. Nature 1998; 395: 451-2.

[2] Canet-Aviles RM, Wilson MA, Miller DW, et al. The Parkinson's disease protein DJ-1 is neuroprotective due to cysteine-sulfinic acid-driven mitochondrial localization. Proc Natl Acad Sci USA 2004; 101: 9103-8.

[3] Kahle PJ, Waak J, Gasser T. DJ-1 and prevention of oxidative stress in Parkinson's disease and other age-related disorders. Free Radic Biol Med 2009; 47: 1354-61. 
[4] Nagakubo D, Taira T, Kitaura H, et al. DJ-1, a novel oncogene which transforms mouse NIH3T3 cells in cooperation with ras. Biochem Biophys Res Commun 1997; 231: 509-13.

[5] Bonifati V, Rizzu P, van Baren MJ, et al. Mutations in the DJ-1 gene associated with autosomal recessive early-onset parkinsonism. Science 2003; 299: 256-9.

[6] Bandopadhyay R, Kingsbury AE, Cookson MR, et al. The expression of DJ-1 (PARK7) in normal human CNS and idiopathic Parkinson's disease. Brain 2004; 127: 420-30.

[7] Galter D, Westerlund M, Belin AC, Olson L. DJ-1 and UCH-L1 gene activity patterns in the brains of controls, Parkinson and schizophrenia patients and in rodents. Physiol Behav 2007; 92: 4653.

[8] Choi J, Sullards MC, Olzmann JA, et al. Oxidative damage of DJ-1 is linked to sporadic Parkinson and Alzheimer diseases. J Biol Chem 2006; 281: 10816-24.

[9] Zhou W, Zhu M, Wilson MA, Petsko GA, Fink AL. The oxidation state of DJ-1 regulates its chaperone activity toward alphasynuclein. J Mol Biol 2006; 356: 1036-48.

[10] Zhang L, Shimoji M, Thomas B, et al. Mitochondrial localization of the Parkinson's disease related protein DJ-1: implications for pathogenesis. Hum Mol Genet 2005; 14: 2063-73.

[11] Hod Y, Pentyala SN, Whyard TC, El-Maghrabi MR. Identification and characterization of a novel protein that regulates RNA-protein interaction. J Cell Biochem 1999; 72: 435-44.

[12] van der Brug MP, Blackinton J, Chandran J, et al. RNA binding activity of the recessive parkinsonism protein DJ-1 supports involvement in multiple cellular pathways. Proc Natl Acad Sci USA 2008; 105: 10244-9.

[13] van Duijn CM, Dekker MC, Bonifati V, et al. Park7, a novel locus for autosomal recessive early-onset parkinsonism, on chromosome 1p36. Am J Hum Genet 2001; 69: 629-34.

[14] Bonifati V, Breedveld GJ, Squitieri F, et al. Localization of autosomal recessive early-onset parkinsonism to chromosome 1p36 (PARK7) in an independent dataset. Ann Neurol 2002; 51: 253-6.
[15] Lockhart PJ, Lincoln S, Hulihan M, et al. DJ-1 mutations are a rare cause of recessively inherited early onset parkinsonism mediated by loss of protein function. J Med Genet 2004; 41: e22.

[16] Nuytemans K, Theuns J, Cruts M, Van BC. Genetic etiology of Parkinson disease associated with mutations in the SNCA, PARK2, PINK1, PARK7, and LRRK2 genes: a mutation update. Hum Mutat 2010; 31: 763-80.

[17] Håkansson A, Belin AC, Stiller C, et al. Investigation of genes related to familial forms of Parkinson's disease--with focus on the Parkin gene. Parkinsonism Relat Disord 2008; 14: 520-2.

[18] Daniel SE, Lees AJ. Parkinson's Disease Society Brain Bank, London: overview and research. J Neural Transm Suppl 1993; 39: 165-72.

[19] Ronaghi M, Uhlen M, Nyren P. A sequencing method based on real-time pyrophosphate. Science 1998; 281: 363-5.

[20] Mathews DH, Sabina J, Zuker M, Turner DH. Expanded sequence dependence of thermodynamic parameters improves prediction of RNA secondary structure. J Mol Biol 1999; 288: 911-40.

[21] Zuker M. Mfold web server for nucleic acid folding and hybridization prediction. Nucleic Acids Res 2003; 31: 3406-15.

[22] Abou-Sleiman PM, Healy DG, Quinn N, Lees AJ, Wood NW. The role of pathogenic DJ-1 mutations in Parkinson's disease. Ann Neurol 2003; 54: 283-6.

[23] Pankratz N, Pauciulo MW, Elsaesser VE, et al. Mutations in DJ-1 are rare in familial Parkinson disease. Neurosci Lett 2006; 408: 209-13.

[24] Lev N, Ickowicz D, Barhum Y, et al. DJ-1 protects against dopamine toxicity. J Neural Transm 2009; 116: 151-60.

[25] Parmley JL, Hurst LD. How do synonymous mutations affect fitness? Bioessays 2007; 29: 515-9.

[26] Bartoszewski RA, Jablonsky M, Bartoszewska S, et al. A synonymous single nucleotide polymorphism in DeltaF508 CFTR alters the secondary structure of the mRNA and the expression of the mutant protein. J Biol Chem 2010; 285: 28741-8.

Received: October 22, 2010

Revised: December 10, 2010

Accepted: January 03, 2011

(C) Anvret et al.; Licensee Bentham Open.

This is an open access article licensed under the terms of the Creative Commons Attribution Non-Commercial License (http://creativecommons.org/licenses/by-nc/3.0/) which permits unrestricted, non-commercial use, distribution and reproduction in any medium, provided the work is properly cited. 